\title{
A Specht Module Analog for the Rook Monoid
}

\author{
Cheryl Grood* \\ Department of Mathematics and Statistics \\ Swarthmore College, Swarthmore, PA, USA \\ cgrood10swarthmore.edu
}

Submitted: July 20, 2001; Accepted: December 18, 2001.

MR Subject Classifications: 05E10, 20M30

\begin{abstract}
The wealth of beautiful combinatorics that arise in the representation theory of the symmetric group is well-known. In this paper, we analyze the representations of a related algebraic structure called the rook monoid from a combinatorial angle. In particular, we give a combinatorial construction of the irreducible representations of the rook monoid. Since the rook monoid contains the symmetric group, it is perhaps not surprising that the construction outlined in this paper is very similar to the classic combinatorial construction of the irreducible $S_{n}$-representations: namely, the Specht modules.
\end{abstract}

\section{Introduction}

Let $R_{n}$ be the set of all $n \times n$ matrices that contain at most one entry of one in each column and row and zeroes elsewhere. Under matrix multiplication, $R_{n}$ has the structure of a monoid, a set with an associative binary operation and an identity element. The monoid $R_{n}$ is known both as the symmetric inverse semigroup and the rook monoid, the latter name stemming from the correspondence between the matrices in $R_{n}$ and the placement of non-attacking rooks on an $n \times n$ chessboard. The number of rank $r$ matrices in $R_{n}$ is $\left(\begin{array}{l}n \\ r\end{array}\right)^{2} r$ ! and hence the rook monoid has a total of $\sum_{r=0}^{n}\left(\begin{array}{l}n \\ r\end{array}\right)^{2} r$ ! elements. Note that the set of rank $n$ matrices in the rook monoid is isomorphic to $S_{n}$, the symmetric group on $n$ letters.

It is often useful to associate each $n \times n$ matrix $\left(a_{i j}\right) \in R_{n}$ with a function $\sigma$, given by

$$
\sigma(j)= \begin{cases}i & \text { if there exists an } i \text { such that } a_{i j}=1 \\ 0 & \text { otherwise. }\end{cases}
$$

${ }^{*}$ The author wishes to thank Stephen Maurer for reading an earlier draft of this paper and giving many useful suggestions. The author is also grateful to Lou Solomon for encouraging her to write up these results and to the referee for several helpful comments. 
This function is well-defined because at most one element in each column is nonzero. For example, with $n=4$ the matrix

$$
\left[\begin{array}{llll}
0 & 0 & 0 & 0 \\
0 & 0 & 1 & 0 \\
1 & 0 & 0 & 0 \\
0 & 0 & 0 & 1
\end{array}\right]
$$

corresponds to a map $\sigma$ that sends 1 to 3,2 to 0,3 to 2 , and 4 to 4 . Note that when $\left(a_{i j}\right)$ is a permutation matrix, the function $\sigma$ is in fact the permutation associated with that matrix. Munn [2] introduced a concise way of representing elements of $R_{n}$ using what he termed cycle-link notation. In this notation, a cycle $\left(a_{1}, a_{2}, \ldots, a_{k}\right)$ means, as in the case of permutations, that

$$
a_{1} \longmapsto a_{2} \longmapsto \cdots \mapsto a_{k} \longmapsto a_{1} .
$$

In contrast, a link $\left[b_{1}, b_{2}, \ldots b_{l}\right]$ means that $b_{1} \mapsto b_{2} \mapsto \cdots b_{l}$ and $b_{l}$ maps to 0 . The $4 \times 4$ matrix above would translate to $[1,3,2](4)$ in cycle-link notation.

Munn's results ([2], [3]) on the representations of rook monoids stemmed from his work on the more general theory of representations of finite semigroups. The representation theory of the rook monoid is particularly interesting because of its similarity to the representation theory of the symmetric group. Munn showed that the complex monoid algebra $\mathbb{C} R_{n}$ is semisimple and subsequently found the irreducible representations of $R_{n}$. He demonstrated that these irreducibles are indexed by partitions of nonnegative integers less than or equal to $n$, whereas the irreducible representations of $S_{n}$ are indexed by partitions of $n$. In addition, Munn computed the characters of the $R_{n}$-irreducibles using irreducible characters of $S_{r}, 1 \leq r \leq n$.

More recently, Solomon ([5], [6]) has investigated $q$-generalizations of the rook monoid as well as an analog of Schur-Weyl duality for $R_{n}$, and Halverson et al.[1] have found an $R_{n}$ analog of the Murnaghan-Nakayama rule, a combinatorial construction of the irreducible characters of $S_{n}$. In this paper, we provide a combinatorial construction for the irreducible representations of $R_{n}$ that is very similar to the Specht module construction of the irreducible representations of $S_{n}$. Our construction and notation closely follow the exposition in [4] on Specht modules.

\section{Preliminaries}

A partition is a finite sequence of nonnegative integers $\lambda=\left(\lambda_{1}, \lambda_{2}, \ldots, \lambda_{m}\right)$ arranged in a weakly decreasing order; that is, $\lambda_{1} \geq \lambda_{2} \geq \ldots \geq \lambda_{m} \geq 0$. The $\lambda_{i}$ are called the parts of the partition. The weight of $\lambda$, denoted $|\lambda|$, is the sum of its parts: $|\lambda|=\sum_{i=1}^{m} \lambda_{i}$. If $|\lambda|=r$, we write: $\lambda \vdash r$. The partition with all parts equal to 0 is the empty partition.

The length of $\lambda$, denoted $\ell(\lambda)$, is the maximum subscript $j$ such that $\lambda_{j}>0$. Associated to each partition $\lambda$ is a Ferrers diagram of shape $\lambda$. The Ferrers diagram has $\lambda_{i}$ boxes in its $i$-th row, and the boxes are left-justified. For example, the Ferrers diagram associated with $\lambda=(6,4,3,2,2,1)$ is 


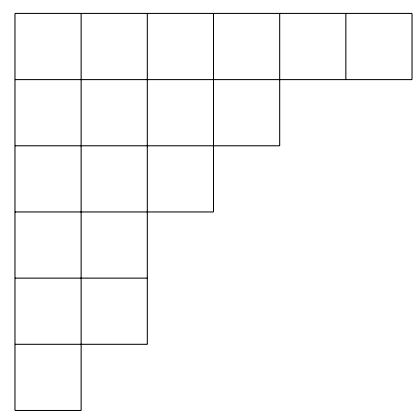

The Ferrers diagram for the empty partition is denoted by $\emptyset$. If $\lambda \vdash r$, we say that a tableau of shape $\lambda$, or a $\lambda$-tableau, is a Ferrers diagram of shape $\lambda$ with the boxes filled with distinct entries from the set $\{1,2, \ldots, r\}$. The following is an example of a tableau of shape $(4,2,1,1)$.

\begin{tabular}{|l|l|l|l|}
\hline 1 & 3 & 4 & 8 \\
\hline 2 & 6 & \multicolumn{2}{|l}{} \\
\cline { 1 - 2 } 5 & \multicolumn{2}{|}{} \\
\cline { 1 - 1 } 7 & \multicolumn{2}{|}{} \\
\cline { 1 - 1 } & &
\end{tabular}

Tableaux are at the core of the Specht module construction for the irreducibles of $S_{n}$. We now introduce a new object which will serve the same function for $R_{n}$ as the tableaux did for $S_{n}$.

Definition 2.1 Let $\lambda \vdash r$, where $0 \leq r \leq n$. An n-tableau of shape $\lambda$, also called a $\lambda_{r}^{n}$-tableau, is a Ferrers diagram of shape $\lambda$ filled with $r$ distinct entries from the set $\{1,2, \ldots, n\}$.

For instance,

\begin{tabular}{|l|l|}
\hline 1 & 5 \\
\hline 3 & 7 \\
\hline 4 & \multicolumn{1}{|c}{} \\
\cline { 1 - 1 } &
\end{tabular}

is an $n$-tableau of shape $(2,2,1)$ for any $n \geq 7$. Note that in the case where $r=n$, an n-tableau of shape $\lambda$ is in fact a tableau of shape $\lambda$. The content of an $\lambda_{r}^{n}$-tableau $t$ is the set of entries in $t$. An $n$-tableau $t$ of shape $\lambda$ is standard if the entries in its rows and columns increase left-to-right and top-to-bottom, respectively.

Notation 2.1 Let $t$ be $a \lambda_{r}^{n}$-tableau. Then $t_{i, j}$ will denote the entry contained in the box of the ith row and $j$ th column of $t$. 
Two $n$-tableaux $t_{1}$ and $t_{2}$ of shape $\lambda$ are row-equivalent if the corresponding rows of the two tableaux contain the same entries; in this case, we write $t_{1} \sim t_{2}$.

Definition 2.2 An n-tabloid $\{t\}$ of shape $\lambda$, or an $\lambda_{r}^{n}$-tabloid $\{t\}$, is the set of all $\lambda_{r}^{n}$ tableaux that are row-equivalent to $t$; i.e.,

$$
\{t\}=\{s \mid s \sim t\} .
$$

Let $N^{\lambda}$ be the vector space over $\mathbb{C}$ generated by the $\lambda_{r}^{n}$-tableaux; that is, $N^{\lambda}$ is the set of all formal $\mathbb{C}$-linear combinations of $\lambda_{r}^{n}$-tableaux. We can define an action of $R_{n}$ on $N^{\lambda}$ by first determining how $R_{n}$ acts on the basis of $\lambda_{r}^{n}$-tableaux and then linearly extending this action to the whole vector space. If $t$ is an $\lambda_{r}^{n}$-tableau, we define $\sigma t$ to equal the zero vector if $t$ contains an entry $t_{i, j}$ such that $\sigma\left(t_{i, j}\right)=0$; when $t$ contains no such entry, we say that $(\sigma t)_{i, j}=\sigma\left(t_{i, j}\right)$. Similarly, if we let $M^{\lambda}$ represent the vector space over $\mathbb{C}$ generated by the $\lambda_{r}^{n}$-tabloids, we have an induced action of $R_{n}$ on $M^{\lambda}$ given by:

$$
\sigma\{t\}= \begin{cases}\mathbf{0} & \text { if } \sigma t=\mathbf{0} \\ \{\sigma t\} & \text { otherwise. }\end{cases}
$$

Since $s \sim t$ implies that $\sigma s \sim \sigma t$, this induced action is well-defined.

Suppose that $t$ is an $\lambda_{r}^{n}$-tableau, and let $C_{i}$ be the entries in the $i$ th column of $t$. We now form the group

$$
C_{t}=S_{C_{1}} \times S_{C_{2}} \times \cdots \times S_{C_{l}}
$$

which we can think of as being contained in $R_{n}$. Each element in $C_{t}$ stabilizes the columns of $t$, but note that $C_{t}$ does not consist of all the elements in $R_{n}$ that fix the columns of $t$. For each $\lambda_{r}^{n}$-tableau $t$, we will generate the following element of $M^{\lambda}$ :

$$
e_{t}=\sum_{\sigma \in C_{t}} \operatorname{sgn}(\sigma) \sigma\{t\}
$$

where sgn stands for the sign of the permutation $\sigma$. We will call $e_{t}$ the $n$-polytabloid associated with $t$. Let $R^{\lambda}$ be the vector space over $\mathbb{C}$ generated by the set of $n$-polytabloids of shape $\lambda$; let $S^{\lambda}$ be the subspace of $R^{\lambda}$ generated by $\left\{e_{t} \mid t, \lambda_{r}^{n}\right.$-tableau; content $t=$ $\{1,2, \ldots, r\}\}$. The subspaces $S^{\lambda}, \lambda \vdash r$, are exactly the so-called Specht modules, a complete set of distinct irreducible $S_{r}$-modules. We will show that the vector spaces $R^{\lambda}$ form a complete set of distinct irreducible $R_{n}$-modules, and hence are analogous to the Specht modules for the symmetric group.

\section{$3 \quad R_{n}$-modules}

The goal of this section is to show that for each $\lambda$ such that $|\lambda| \leq n, R^{\lambda}$ is an $R_{n}$-module. For that, we will need some new notation and several lemmas.

Definition 3.1 Let $\pi \in R_{n}$. Then $\widehat{\pi}$ will denote the element in $S_{n}$ that comes from changing all the links in $\pi$ to cycles. 
For example, if $\pi=[1,4,6](2,3)[5]$, then $\widehat{\pi}=(1,4,6)(2,3)(5)$. If $\pi \in S_{n}$, then clearly $\pi=\widehat{\pi}$.

Lemma 3.1 Suppose $\pi \in R_{n}$ and $t$ is a $\lambda_{r}^{n}$-tableau. If $\pi t \neq \mathbf{0}$, then $\pi t=\widehat{\pi} t$.

Proof. Express $\pi$ in cycle-link notation. Since $\pi t \neq \mathbf{0}$, none of the entries $t_{i, j}$ occur as a right-most element of a link of $\pi$. Thus $\pi\left(t_{i, j}\right)=\widehat{\pi}\left(t_{i, j}\right)$ for all $i, j$ and so $\pi t=\widehat{\pi} t$.

Lemma 3.2 If $\sigma \in S_{n}$ and $t$ is an $\lambda_{r}^{n}$-tableau with column entries $C_{1}, \ldots, C_{l}$, then $C_{\sigma t}=$ $\sigma C_{t} \sigma^{-1}$.

Proof. Let $\pi=\left(i_{1}, i_{2}, \ldots, i_{j}\right)\left(i_{j+1}, \ldots, i_{k}\right) \cdots\left(i_{m}, \ldots, i_{n}\right)$ be an element in $S_{n}$ expressed in cycle notation. Now consider $\sigma C_{t} \sigma^{-1}$. If we view $C_{t}$ as a subgroup of $S_{n}$, it follows that $\sigma C_{t} \sigma^{-1}=\sigma S_{C_{1}} \sigma^{-1} \times \cdots \times \sigma S_{C_{l}} \sigma^{-1}$. Because

$$
\sigma \pi \sigma^{-1}=\left(\sigma\left(i_{1}\right), \sigma\left(i_{2}\right), \ldots, \sigma\left(i_{j}\right)\right)\left(\sigma\left(i_{j+1}\right), \ldots, \sigma\left(i_{k}\right)\right) \cdots\left(\sigma\left(i_{m}\right), \ldots, \sigma\left(i_{n}\right)\right),
$$

it follows that $\sigma S_{C_{i}} \sigma^{-1}=S_{\sigma\left(C_{i}\right)}$, where $\sigma\left(C_{i}\right)$ represents the set of elements $\{\sigma(j) \mid j \in$ $\left.C_{i}\right\}$. So,

$$
\sigma C_{t} \sigma^{-1}=S_{\sigma\left(C_{1}\right)} \times \cdots \times S_{\sigma\left(C_{l}\right)},
$$

which is exactly $C_{\sigma t}$.

These lemmas allow us to prove the following proposition.

Proposition 3.3 Suppose $\pi \in R_{n}, t$ is a $\lambda_{r}^{n}$-tableau. If $\pi t=\mathbf{0}$, then $\pi e_{t}=\mathbf{0}$. Otherwise, $\pi e_{t}=e_{\widehat{\pi} t}$.

Proof. First consider the case where $\pi t=\mathbf{0}$. Recall that $\pi t=\mathbf{0}$ if and only if $\pi\{t\}=\mathbf{0}$ as well. Each term in the linear combination of tabloids that form $e_{t}$ contains the exact same entries as $t$. Therefore, if $\pi\{t\}=\mathbf{0}$, then $\pi\{s\}=\mathbf{0}$ for all $\{s\}$ in the linear combination $e_{t}$; it follows that in this case, $\pi e_{t}=\mathbf{0}$.

If $\pi t \neq \mathbf{0}$, we have $\pi t=\widehat{\pi} t$ by Lemma 3.1. Thus, $\pi\{s\}=\widehat{\pi}\{s\}$ for every tabloid $\{s\}$ that appears in the linear combination $e_{t}$, and hence $\pi e_{t}=\widehat{\pi} e_{t}$. By manipulating the terms of $\widehat{\pi} e_{t}$, we can conclude that

$$
\begin{aligned}
\pi e_{t} & =\widehat{\pi} e_{t} \\
& =\widehat{\pi} \sum_{\sigma \in C_{t}} \operatorname{sgn}(\sigma) \sigma\{t\} \\
& =\sum_{\sigma \in C_{t}} \operatorname{sgn}(\sigma) \widehat{\pi} \sigma\{t\} \\
& =\sum_{\sigma \in C_{t}} \operatorname{sgn}\left(\widehat{\pi} \sigma \widehat{\pi}^{-1}\right) \widehat{\pi} \sigma \widehat{\pi}^{-1} \widehat{\pi}\{t\} \\
& =\sum_{\sigma \in C_{t}} \operatorname{sgn}\left(\widehat{\pi} \sigma \widehat{\pi}^{-1}\right) \widehat{\pi} \sigma \widehat{\pi}^{-1}\{\widehat{\pi} t\}
\end{aligned}
$$




$$
\begin{aligned}
& =\sum_{\gamma \in \widehat{\pi} C_{t} \widehat{\pi}^{-1}} \operatorname{sgn}(\gamma) \gamma\{\widehat{\pi} t\} \\
& =\sum_{\gamma \in C_{\widehat{\pi} t}} \operatorname{sgn}(\gamma) \gamma\{\widehat{\pi} t\} \quad \text { (by Lemma 3.2) } \\
& =e_{\widehat{\pi} t} .
\end{aligned}
$$

Thus, by Proposition 3.3, it follows that for any $\pi \in R_{n}$ and $e_{t}$ in $R^{\lambda}, \pi e_{t}$ equals either 0 or $e_{\widehat{\pi} t}$, both of which are again elements of $R^{\lambda}$. This immediately gives us the following corollary.

Corollary $3.4 R^{\lambda}$ is an $R_{n}$-module.

Furthermore, we are able to show easily that

Corollary $3.5 R^{\lambda}$ is a cyclic $R_{n}$-module.

Proof. Note that if $s$ and $t$ are both $\lambda_{r}^{n}$-tableaux, there exists an element $\sigma$ in $S_{n}$ such that $s=\sigma t$. By Proposition 3.3, we have that $\sigma e_{t}=e_{\widehat{\sigma} t}$. Since $\sigma \in S_{n}, \sigma=\widehat{\sigma}$, and so $\sigma e_{t}=e_{\sigma t}$, which in turn equals $e_{s}$. Since we can generate all the basis elements of $R^{\lambda}$ using one element $e_{t}, R^{\lambda}$ is cyclic.

\section{Irreducibility of $R^{\lambda}$}

Let $|\lambda|=r$, with $r \leq n$. Recall that the subspace $S^{\lambda}$ of $R^{\lambda}$ is an irreducible $S_{r}$-module. We will use this fact to help show that $R^{\lambda}$ is an irreducible $R_{n}$-module.

Theorem 4.1 If $|\lambda| \leq n, R^{\lambda}$ is an irreducible $R_{n}$-module.

Proof. Let $U$ be a nonzero submodule of $R^{\lambda}$. We need to show that $U$ must in fact be equal to $R^{\lambda}$. Since $R^{\lambda}$ is a cyclic module, it suffices to show that we can find a $\lambda_{r}^{n}$-tableau $t$ such that $e_{t} \in U$.

Let $\mathbf{u}$ be a nonzero element of $U$, and write $\mathbf{u}$ as a linear combination of n-polytabloids:

$$
\mathbf{u}=\sum_{t, \lambda_{r}^{n} \text {-tableau }} a_{t} e_{t}
$$

We now group the tabloids based on their content; i.e.,

$$
\mathbf{u}=\sum_{\substack{X \subset\{1, \ldots, n\} \\|X|=r}}\left(\sum_{t, \text { tableau of content } X} a_{t} e_{t}\right)
$$


Since $\mathbf{u} \neq \mathbf{0}$, there must be at least one set $X_{0}$ such that

$$
\sum_{t, \text { content } t=X_{0}} a_{t} e_{t} \neq \mathbf{0} .
$$

If $X_{0}=\left\{i_{1}, i_{2}, \ldots, i_{r}\right\}$, where $i_{1}<i_{2}<\ldots<i_{r}$, consider the element $\pi$ in $R_{n}$ defined by

$$
\pi(k)= \begin{cases}0 & \text { if } k \notin X_{0} \\ j & \text { if } k=i_{j} .\end{cases}
$$

Note that we have defined $\pi$ in such a way so that $\pi t=0$ if and only if content $t \neq X_{0}$. Multiplying $\mathbf{u}$ by $\pi$, we obtain

$$
\pi \mathbf{u}=\sum_{t, \lambda_{r}^{n} \text {-tableau }} a_{t}\left(\pi e_{t}\right)
$$

By Proposition 3.3, if $\pi t=\mathbf{0}$, then $\pi e_{t}=\mathbf{0}$. We have defined $\pi$ in such a way that all the terms in $\pi \mathbf{u}$ with content not equal to $X_{0}$ vanish. Thus, $\pi \mathbf{u}$ simplifies to

$$
\pi \mathbf{u}=\sum_{t, \text { content } t=X_{0}} a_{t}\left(\pi e_{t}\right),
$$

which in turn equals $\quad \sum \quad a_{t}\left(\widehat{\pi} e_{t}\right)$, since both $\pi$ and $\widehat{\pi}$ act the same on tabloids $t$, content $t=X_{0}$

of content $X_{0}$. Multiplying on the left by $\widehat{\pi}^{-1}$, we see that

$$
\begin{aligned}
\mathbf{u} & =\widehat{\pi}^{-1}(\pi \mathbf{u}) \\
& =\sum_{t, \text { content } t=X_{0}} a_{t} \widehat{\pi}^{-1}\left(\widehat{\pi} e_{t}\right) \\
& =\sum_{t, \text { content } t=X_{0}} a_{t} e_{t},
\end{aligned}
$$

which is nonzero, so $\pi \mathbf{u}$ cannot be $\mathbf{0}$.

Now we apply Proposition 3.3 to (1), so that

$$
\pi \mathbf{u}=\sum_{t, \text { content } t=X_{0}} a_{t} e_{\widehat{\pi} t} .
$$

Now observe that when the content of $t$ is $X_{0}$, the content of $\pi t$ equals $\{1,2, \ldots, r\}$, which in turn implies that $e_{\widehat{\pi} t} \in S^{\lambda}$. Since $\pi \mathbf{u}$ is a linear combination of elements in $S^{\lambda}, \pi \mathbf{u}$ itself is an element of $S^{\lambda}$. Hence $U \cap S^{\lambda} \neq \mathbf{0}$, since the intersection contains the nonzero element $\pi \mathbf{u}$. Viewing $U$ as an $S_{r}$-module (by realizing $S_{r} \subset R_{n}$ ), we have that $U \cap S^{\lambda}$ is a nonzero

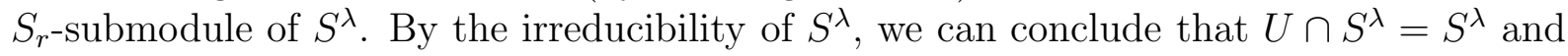
thus $S^{\lambda} \subseteq U$. Now pick any $e_{t} \in S^{\lambda} ; e_{t}$ must also be contained in $U$, and since $R^{\lambda}$ is cyclic, we have that $U=R^{\lambda}$ as desired.

Now that we know that these modules are irreducible, we need to show that they are all distinct. 
Theorem 4.2 If $R^{\lambda}$ and $R^{\mu}$ are isomorphic as $R_{n}$-modules, then $\lambda=\mu$.

Proof. Let $\Theta: R^{\lambda} \longrightarrow R^{\mu}$ be an $R_{n}$-isomorphism. Let $t$ be a $\lambda_{r}^{n}$-tableau. The image of $e_{t}$ under $\Theta$ is a linear combination of $\left\{e_{s} \mid s\right.$, standard $\mu_{r}^{n}$-tableaux $\}$. If the entry $i$ is in $t$, then $\pi=(1)(2) \cdots \widehat{(i)} \cdots(n)[i]$ annihilates $t$, and hence $e_{t}$ as well. Thus, $\Theta\left(\pi e_{t}\right)=\mathbf{0}$, which implies that

$$
\begin{aligned}
& \mathbf{0}=\pi \Theta\left(e_{t}\right) \\
& =\pi\left(\sum_{s, \text { standard } \mu_{r}^{n}-\text { tableau }} a_{s} e_{s}\right) \\
& =\sum_{s, \text { standard } \mu_{r}^{n}-\text { tableau }} a_{s} \pi e_{s} \\
& =\quad \sum \quad a_{s} e_{\widehat{\pi}_{s}} \quad \text { (by Proposition 3.3) } \\
& s \text {, standard } \mu_{r}^{n}-\text { tableau } \\
& \pi s \neq \mathbf{0} \\
& =\sum_{\substack{s, \text { standard } \mu_{r}^{n}-\text { tableau } \\
\pi s \neq \mathbf{0}}} a_{s} e_{s},
\end{aligned}
$$

since $\widehat{\pi}$ is the identity element. The only linear combination of basis elements that equals $\mathbf{0}$ is the trivial one; thus, $\Theta\left(e_{t}\right)$ is a linear combination of $\left\{e_{s} \mid s\right.$, standard $\mu_{r}^{n}$-tableau, $\pi s=$ $\mathbf{0}$ \}. However, the only tableaux that $\pi$ annihilates are those that contain an entry of $i$. Since this argument holds for every $i$ in the content of $t, \Theta\left(e_{t}\right)$ is a linear combination of $\mu_{r}^{n}$-polytabloids $e_{s}$ such that the content of $t$ is a subset of the content of $s$. Since $\Theta$ is invertible, we in fact have that

$$
\Theta\left(e_{t}\right)=\sum_{\substack{s, \text { standard } \mu_{r}^{n}-\text { tableau } \\ \text { content } s \stackrel{\text { content } t}{=}}} a_{s} e_{s} .
$$

Recall that $S^{\lambda}$ is the subspace of $R^{\lambda}$ generated by the polytabloids with content $1,2, \ldots,|\lambda|$. It follows from Equation 2 that the image of $S^{\lambda}$ under $\Theta$ is contained in $S^{\mu}$; that is, $S^{\lambda}$ is isomorphic to a submodule of $S^{\mu}$. The only way this can occur is if $\lambda=\mu$, which is what we wanted to show.

\section{$5 \quad$ A Basis for $R^{\lambda}$}

In closing, we give a combinatorial basis for our irreducible $R_{n}$-modules $R^{\lambda}$. Again, the story closely resembles what happens in the case of the irreducible $S_{r}$-modules $S^{\lambda}$. In that case, a basis for $S^{\lambda}$ consists of $\left\{e_{t} \mid t\right.$ is a standard tableau of shape $\left.\lambda\right\}$. We will establish that a similar statement holds for $R^{\lambda}$ : 
Theorem 5.1 Let $\lambda \vdash r$, with $0 \leq r \leq n$. Then the set

$$
\left\{e_{t} \mid t \text { is a standard } \lambda_{r}^{n} \text {-tableau }\right\}
$$

is a basis for $R^{\lambda}$.

Proof. First, we will show that the $e_{t}$ are linearly independent. If the content of two $\lambda_{r}^{n}$-tableaux $s$ and $t$ differ, it is not hard to see that $e_{t}$ and $e_{s}$ are independent. Therefore, it suffices to show that

$$
\left\{e_{t} \mid t \text {, standard } \lambda_{r}^{n} \text {-tableau with content } X\right\}
$$

is independent. Without loss of generality, assume that the content $X$ is the set $\{1,2, \ldots, r\}$. Since these polytabloids form a basis for $S^{\lambda}$, they are independent, which is what we wanted to show.

Next, we compute the order of $\left\{e_{t} \mid t\right.$ is a standard $\lambda_{r}^{n}$-tableau $\}$. There are $\left(\begin{array}{l}n \\ r\end{array}\right)$ ways to choose a fixed content $X$. Let $f_{\lambda}$ denote the number of standard tableaux of shape $\lambda$. Then in fact $f_{\lambda}$ counts the number of $\lambda_{r}^{n}$-tableaux with a given content $X$. Hence, the set $\left\{e_{t} \mid t\right.$ is a standard $\lambda_{r}^{n}$-tableau $\}$ has $\left(\begin{array}{l}n \\ r\end{array}\right) f_{\lambda}$ elements. Since we have shown that these vectors are independent, we can conclude that the dimension of $R^{\lambda}$ is at least $\left(\begin{array}{l}n \\ r\end{array}\right) f^{\lambda}$.

For semisimple algebras, the sum of the squares of the dimensions of its nonisomorphic irreducible modules equals the dimension of the algebra itself; i.e.,

$$
\sum_{I, \text { irreducible } A-\text { module }}(\operatorname{dim} I)^{2}=\operatorname{dim} A .
$$

Suppose there is some $\lambda$ such that the dimension of $R^{\lambda}$ is strictly larger than $\left(\begin{array}{l}n \\ r\end{array}\right) f^{\lambda}$. Then we would have

$$
\begin{aligned}
\sum_{r=0}^{n}\left(\begin{array}{l}
n \\
r
\end{array}\right)^{2} r ! & =\operatorname{dim} \mathbb{C} R_{n} \\
& <\sum_{r=0}^{n} \sum_{\lambda \vdash r}\left(\operatorname{dim} R^{\lambda}\right)^{2} \\
& =\sum_{r=0}^{n} \sum_{\lambda \vdash r}\left(\begin{array}{l}
n \\
r
\end{array}\right)^{2}\left(f_{\lambda}\right)^{2} \\
& =\sum_{r=0}^{n}\left(\begin{array}{l}
n \\
r
\end{array}\right)^{2}\left(\sum_{\lambda \vdash r}\left(f_{\lambda}\right)^{2}\right) .
\end{aligned}
$$

But, it is a well-known combinatorial identity that

$$
\sum_{\lambda \vdash r}\left(f_{\lambda}\right)^{2}=r !
$$

(cf. [4]), and so every irreducible module $R^{\lambda}$ must have dimension equal to $\left(\begin{array}{l}n \\ r\end{array}\right) f^{\lambda}$. Hence, the set of independent vectors $\left\{e_{t} \mid t\right.$ is a standard $\lambda_{r}^{n}$-tableau $\}$ also spans $R^{\lambda}$. Thus we have shown that $\left\{e_{t} \mid t\right.$ is a standard $\lambda_{r}^{n}$-tableau $\}$ is a basis for $R^{\lambda}$. 


\section{References}

[1] M. Dieng, T. Halverson, and V. Poladian, Characters of the q-rook monoid, preprint, 2001.

[2] W.D. Munn, Matrix representations of semigroups, Proc. Camb. Phil. Soc., 53 (1957), 5-12.

[3] W.D. Munn, The characters of the symmetric inverse semigroup, Proc. Camb. Phil. Soc., 53 (1957), 13-18.

[4] B. Sagan, The Symmetric Group, Wadsworth and Brooks Cole, Pacific Grove, CA, 1991.

[5] L. Solomon, Representations of the Rook Monoid, to appear in J. Algebra.

[6] L. Solomon, Representations of the Symmetric Inverse Semigroup, Address given at C.R.M. Montréal, 1997. 